\title{
POINT CLOUD SMOOTH SAMPLING AND SURFACE RECONSTRUCTION BASED ON MOVING LEAST SQUARES
}

\author{
Chuanli Kang ${ }^{1,2}$, Tuanneng $\mathrm{Lu}^{1,2}{ }^{*}$, Mingming Zong ${ }^{1,2}$, Wang Fen ${ }^{1,2}$, Cheng Yao ${ }^{1,2}$ \\ ${ }^{1}$ Guangxi Key Laboratory of Spatial Information and Geomatics, Guilin 541006, China; \\ ${ }^{2}$ College of Geomatics and Geoinformation, Guilin University of Technology, Guilin 541006.China-1099857450@qq.com
}

\section{Commission II, WG II/3}

KEY WORDS: Moving Least Squares, Point Cloud, Smooth Sampling, Voxel Grid Sampling, Uniform Sampling, Surface Reconstruction

\begin{abstract}
:
In point cloud data processing, smooth sampling and surface reconstruction are important aspects of point cloud data processing. In view of the current point cloud sampling method, the point cloud distribution is not uniform, the point cloud feature information is incomplete, and the reconstructed model surface is not smooth. This paper proposes a method of smoothing sampling processing and surface reconstruction using point cloud using moving least squares method. This paper first introduces the traditional moving least squares method in detail, and then proposes an improved moving least squares method for point cloud smooth sampling and surface reconstruction. In this paper, the algorithm is designed for the proposed theory, combined with $\mathrm{C}++$ and point cloud library PCL programming, using voxel grid sampling and uniform sampling and moving least squares smooth sampling comparison, after sampling, using greedy triangulation algorithm surface reconstruction. The experimental results show that the improved moving least squares method performs point cloud smooth sampling more uniformly than the voxel grid sampling and the feature information is more prominent. The surface reconstructed by the moving least squares method is smooth, the surface reconstructed by the voxel grid sampling and the uniformly sampled data surface is rough, and the surface has a rough triangular surface. Point cloud smooth sampling and surface reconstruction based on moving least squares method can better maintain point cloud feature information and smooth model smoothness. The superiority and effectiveness of the method are demonstrated, which provides a reference for the subsequent study of point cloud sampling and surface reconstruction.
\end{abstract}

* Corresponding author: Tuanneng Lu; Email: 1099857450@qq.com 


\section{INTRODUCTION}

With the rapid development of modern scanning technology, $3 \mathrm{D}$ reconstruction technology has been widely used in various industry fields. With the rapid development of modern scanning technology, 3D reconstruction technology has been widely used in various industries, such as reverse engineering (Kang, 2018) ( Zhu et al., 2017), virtual reality (Liang, 2018) ( Wang, 2018), machine vision (Shi, 2017) and other fields. The original point cloud data scanned by the instrument usually contains a large number of noise points and a huge amount of data. If these raw data are directly used for surface reconstruction, the reconstructed surface will be not smooth or vulnerable, and the desired surface model will not be obtained. Moreover, the huge amount of data will put higher demands on the computer in terms of storage and display, resulting in inefficient data processing in the later stage. Therefore, under the premise of retaining the details of point cloud data and not affecting the accuracy of model reconstruction, point cloud reduction is required for massive data (Huang et al., 2018) (Zhai, 2015) (Chen, 2011), so as to achieve the purpose of removing redundant points and improving reconstruction efficiency. Many scholars at home and abroad have done a lot of research on sampling and surface reconstruction of point cloud data. Literature ( $\mathrm{Li}, 2016)$, the curvature is used as the basis of point cloud data processing. An improved Meyer algorithm is proposed to improve the accuracy of curvature estimation. The feature area and the flat area are divided based on the curvature value, and then the curvature sampling and uniform sampling processing are used respectively, achieve the effect of reducing point clouds and retaining the details of the model. However, this method is more difficult to determine the division of the average curvature of each data point with the specified average curvature value, and is not suitable for flat area point clouds. Literature (Bernard et al., 2017) proposed a statistical shape model using the associated point distribution model, but due to the heteroscedasticity of the point cloud data, the surface generated by the surface reconstruction method using only the probabilistic model will have a certain deviation. Literature (Ma et al., 2017), the traditional least squares method is used for plane fitting. Although this algorithm is easy to implement, it is greatly affected by noise points and discrete group points, and the point cloud data is heteroscedastic. Therefore, there is a certain error in calculating the curvature using the traditional least squares method. Literature (Tang et al., 2019), a point cloud reduction algorithm based on weighted least squares curvature calculation is proposed. The algorithm is better in a relatively flat area, but retains more feature points for uneven areas. This method is less effective. Due to various defects in the above-mentioned various algorithms, the point cloud data feature point information after sampling is relatively small, and the reconstructed surface is not smooth or has a loophole. Based on this, this paper proposes a point cloud smooth sampling and surface reconstruction based on moving least squares method.

\section{PRINCIPLE OF MOVING LEAST SQUARES}

The moving least squares method is an optimization method of the least squares method, which has the advantage of high precision and can solve the practical problems that the least squares method cannot solve. Compared with the traditional least squares method, the moving least squares method has two major improvements (Zeng et al., 2004): the establishment of a fitting function is different. This method establishes the fitting function instead of using a traditional polynomial or other function, but consists of a coefficient vector $\mathrm{a}(\mathrm{x})$ and a basis function $\mathrm{p}(\mathrm{x})$, where $\mathrm{a}(\mathrm{x})$ is not a constant but a function of the coordinate $\mathrm{x}$. Second, the concept of compact support is introduced in the moving least squares method. It is considered that the value $\mathrm{y}$ at the point $\mathrm{x}$ is only affected by the nodes in the subdomain near $\mathrm{x}$. This sub-domain is called the influence area of point $\mathrm{x}$, and the nodes outside the influence area have no influence on the value of $\mathrm{x}$. Define a weight function on the affected area $w(x)$. If the weight function is constant over the entire area, the traditional least squares method is obtained. The weight function is an important parameter when moving the least squares method. Whether the curve is smooth or not depends on the choice of the weight function, and the accuracy of the fitting is largely affected by the order of the basis function (Huang et al., 2010) (Guo, 2016).

\subsection{Establishment of a fitting function}

On a local subdomain of the fitted region, the fitting function $f(x)$ is expressed as:

$$
\mathrm{f}(x)=\sum_{i=1}^{m} \alpha_{i}(x) p_{i}(x)=p^{T}(x) \alpha(x)
$$

Where, $\alpha(x)=\left[a_{1}(x), a_{2}(x), \cdots a_{m}(x)\right]^{T}$ for the coefficient 
to be obtained, it is a function of the coordinate $\mathrm{x}$. $p(x)=\left[p_{1}(x), p_{2}(x), \ldots p_{m}(x)\right]^{T}$ for the basis function, it is a k-order complete polynomial, and $\mathrm{m}$ is the number of terms of the basis function. For a one-dimensional problem, the basis function can be:

$p(x)=\left[1, x, x^{2}, \cdots, x^{m}\right]$

For two-dimensional problems, the linear basis is:

$p(x)=[1, x, y]^{T}, m=3$

Secondary basis:

$p(x)=\left[1, x, y, x^{2}, x y, y^{2}\right]^{T}, m=6$

In order to obtain a more accurate local approximation as much as possible, it is necessary to minimize the squared weight of the difference between the local approximation $f\left(x_{i}\right)$ and the node value $y_{i}$. Therefore, the discrete weighted paradigm of the residual $L_{2}$ (Chen et al., 2013) is:

$J=\sum_{i=1}^{N} w\left(x-x_{i}\right)\left[f(x)-y_{i}\right]^{2}=\sum_{i=1}^{N} w\left(x-x_{i}\right)\left[p^{T}\left(x_{i}\right) \alpha(x)-y_{i}\right]^{2}$

$J=(P \alpha(x)-Y)^{T} W(x)(P \alpha(x)-Y)$

Where

$Y=\left(y_{1}, y_{2}, \cdots, y_{n}\right)^{T}$

(7)

$$
p(x)=\left[\begin{array}{cccc}
p_{1}\left(x_{1}\right) & p_{2}\left(x_{1}\right) & \cdots & p_{m}\left(x_{1}\right) \\
p_{1}\left(x_{2}\right) & p_{2}\left(x_{2}\right) & \cdots & p_{m}\left(x_{2}\right) \\
\vdots & \vdots & \ddots & \vdots \\
p_{1}\left(x_{n}\right) & p_{2}\left(x_{n}\right) & \cdots & p_{m}\left(x_{n}\right)
\end{array}\right]
$$

$w(x)=\operatorname{diag}\left[w\left(x-x_{1}\right), w\left(x-x_{2}\right), \cdots, w\left(x-x_{n}\right)\right]$

Where $\mathrm{N}$ is the number of nodes in the solution region and $f(x)$ is the fitting function, $w\left(x-x_{i}\right)$ which is the weight function of the node $x_{i}$. The introduction of the tight support weight function $w(x)=w\left(x-x_{i}\right)$ effectively solves the defect that the piecewise fitting cannot be localized, and can fully consider the influence of each node on the point to be fitted. The weight function is only related to the distance between the node and the point to be fitted. It is centered on the i-th node and has a value only in the neighborhood around the i-th node.
In the moving least squares algorithm, the coefficients $\alpha_{i}(x)$ are chosen such that the fitting function $\mathrm{f}(\mathrm{x})$ finds the best approximation in the neighborhood of the computed point $\mathrm{x}$. To determine the coefficient, let $\mathrm{J}$ take the minimum value, for any function $h(x)$ and $g(x)$

$(h, g)=\sum_{i=1}^{n} w\left(x-x_{i}\right) h\left(x_{i}\right) g\left(x_{i}\right)$

$\alpha_{1}(x)\left(p_{i}, p_{1}\right)+\alpha_{2}(x)\left(p_{i}, p_{2}\right)+\cdots+\alpha_{m}(x)\left(p_{i}, p_{m}\right)=\left(p_{i}, y_{i}\right)$

$i=1,2, \cdots m$

Written in matrix form, you can get the system of equations:

$\left[\begin{array}{cccc}\left(p_{1}, p_{1}\right) & \left(p_{1}, p_{2}\right) & \cdots & \left(p_{1}, p_{m}\right) \\ \left(p_{2}, p_{1}\right) & \left(p_{2}, p_{2}\right) & \cdots & \left(p_{2}, p_{m}\right) \\ \vdots & \vdots & \ddots & \vdots \\ \left(p_{m}, p_{1}\right) & \left(p_{m}, p_{2}\right) & \cdots & \left(p_{m}, p_{m}\right)\end{array}\right]\left[\begin{array}{c}\alpha_{1}(x) \\ \alpha_{2}(x) \\ \vdots \\ \alpha_{m}(x)\end{array}\right]=\left[\begin{array}{c}\left(p_{1}, y_{i}\right) \\ \left(p_{2}, y_{i}\right) \\ \vdots \\ \left(p_{m}, y_{i}\right)\end{array}\right]$

The solution of the above equations can be obtained $\alpha(x)$

$A=\left[\begin{array}{cccc}\left(p_{1}, p_{1}\right) & \left(p_{1}, p_{2}\right) & \cdots & \left(p_{1}, p_{m}\right) \\ \left(p_{2}, p_{1}\right) & \left(p_{2}, p_{2}\right) & \cdots & \left(p_{2}, p_{m}\right) \\ \vdots & \vdots & \ddots & \vdots \\ \left(p_{m}, p_{1}\right) & \left(p_{m}, p_{2}\right) & \cdots & \left(p_{m}, p_{m}\right)\end{array}\right]$

$\alpha=\left[\begin{array}{c}\alpha_{1}(x) \\ \alpha_{2}(x) \\ \vdots \\ \alpha_{m}(x)\end{array}\right] \quad, \quad B y=\left[\begin{array}{c}\left(p_{1}, y_{i}\right) \\ \left(p_{2}, y_{i}\right) \\ \vdots \\ \left(p_{m}, y_{i}\right)\end{array}\right]$

$A=p^{T} w p \quad, \quad B=p^{T} w$

Then formula (12) can be written as:

$A \alpha=B y$

$\alpha=A^{-1} B y$

Bring the (1) formula to get the MLS fitting function:

$f(x)=\sum_{i=1}^{n} \phi_{i}(x) y_{i}$

Where the shape function:

$\phi_{i}(x)=\sum_{j=1}^{m} p_{j}(x)\left(A^{-1} B\right)$

\subsection{Tight support weight function}

The weight function has an extremely important role and significance for the least squares fitting. The weight function $w\left(x-x_{i}\right)$ in the moving least squares method should have tightness, that is, the weight function does not equal 0 in a 
subfield of $\mathrm{x}$, and is 0 outside this subfield. This subdomain is called the support domain of the weight function (that is, the influence domain of x), and its radius is recorded as $S_{\max }$. Here are a few guidelines to follow when choosing a weight function:

(1) The tight support of the weight function determines that the weight at each node is greater than 0 in the support domain and equal to zero outside the support region or at the boundary.

(2) Must have unit decomposition.

(3) The weight function should be smooth and continuous, and the function can be derived.

(4) When in the support domain, the weight decreases as the distance from the node increases.

According to the conditions satisfied by the above weight function, the weight function $w\left(x-x_{I}\right)$ with tight support indicates that only the nodes contained in the support domain to be fitted $\mathrm{x}$ have an effect on $\mathrm{x}$; the weight function $w\left(x-x_{I}\right)$ is non-negative and monotonically decreasing as the distance increases. A commonly used weight function is a cubic spline weight function whose expression is:

$$
w(s)=\left\{\begin{array}{cl}
\frac{2}{3}-4 s^{-2}+4 s^{-3} & (\bar{s}) \leq \frac{1}{2} \\
\frac{4}{3}-4 s^{-1}+4 s^{-2}-\frac{4}{3} s^{-3} & \left(\frac{1}{2}<\bar{s} \leq 1\right) \\
0 & (\bar{s}>1)
\end{array}\right.
$$

Where $\bar{s}$ is the relative amount of distance and $s=x-x_{I}$ is the distance from the point to the point of the node

\subsection{Improved moving least squares method}

When the fitting curve passes through certain nodes, but the distance between the nodes is large, if the traditional moving least squares method is used for fitting directly, deviation may occur. In this paper, the proposed moving least squares method with value added can better reduce the impact of this deviation.

Assuming that the discrete group points are $\left(x_{i}, y_{i}\right)$, $i=1,2, \cdots, n$ and the value-added condition is $\left(x_{s}, y_{s}\right), s=1,2, \cdots, t, t \leq n$, using the general moving least squares method to obtain the fitted curve, the curve with the added value of moving least squares is expressed as:

$$
y=f(x)-\sum_{s=1}^{t} h_{s}(x) \gamma_{s}
$$

Where,

$h_{s}(x)=\prod_{j=1, s \neq j}^{t} \frac{\left(x-x_{j}\right)}{\left(x_{s}-x_{j}\right)}, s=1,2, \cdots, t, \gamma_{s}=f\left(x_{s}\right)-y_{s}$

Basic idea of the algorithm:

(1) Determine the discrete group points $\left(x_{i}, y_{i}\right), i=1,2, \cdots, n$ calculate and store the corresponding normal of the point cloud. (2) Define a circle in the plane neighborhood of each sample point, project the circle onto the fitted surface, and determine the radius of the search neighborhood.

(3) Set the value-added radius so that it meets the value-added condition $\left(x_{s}, y_{s}\right), s=1,2, \cdots, t, t \leq n$, and then add a point cloud in a certain direction.

(4) Since the added point cloud cannot determine its direction, the algorithm takes the sample point as the center and iteratively adds value to the surrounding, setting the number of iterations.

\section{EXPERIMENT AND ANALYSIS}

The algorithm in this paper is configured on the PC as Intel(R) Core(TM) i5-4590 CPU, running at $3.3 \mathrm{GHz}$, RAM at $8.0 \mathrm{G}$, running on Window $764 \mathrm{~W}$ operating system. Experimental research was carried out under the development tool VS2015 and the point cloud library PCL1.8.0. The experiment used RIEGL VZ-1000 high-precision ground 3D laser scanner to collect data from the school motto. The vertical resolution of the scan was $0.015^{\circ}$ and the horizontal resolution was $0.02^{\circ}$. Since it is a verification algorithm, in order to reduce the experimental time, this paper selects one of the scanning station data as the experimental sample data. The sample data is trimmed to obtain the original data of the experiment, as shown in Figure 1. The experiment uses the commonly used voxel grid sampling and uniform sampling methods to sample the original data and compare it with the MLS smooth sampling. The experimental results are shown in Figure 2-4: 


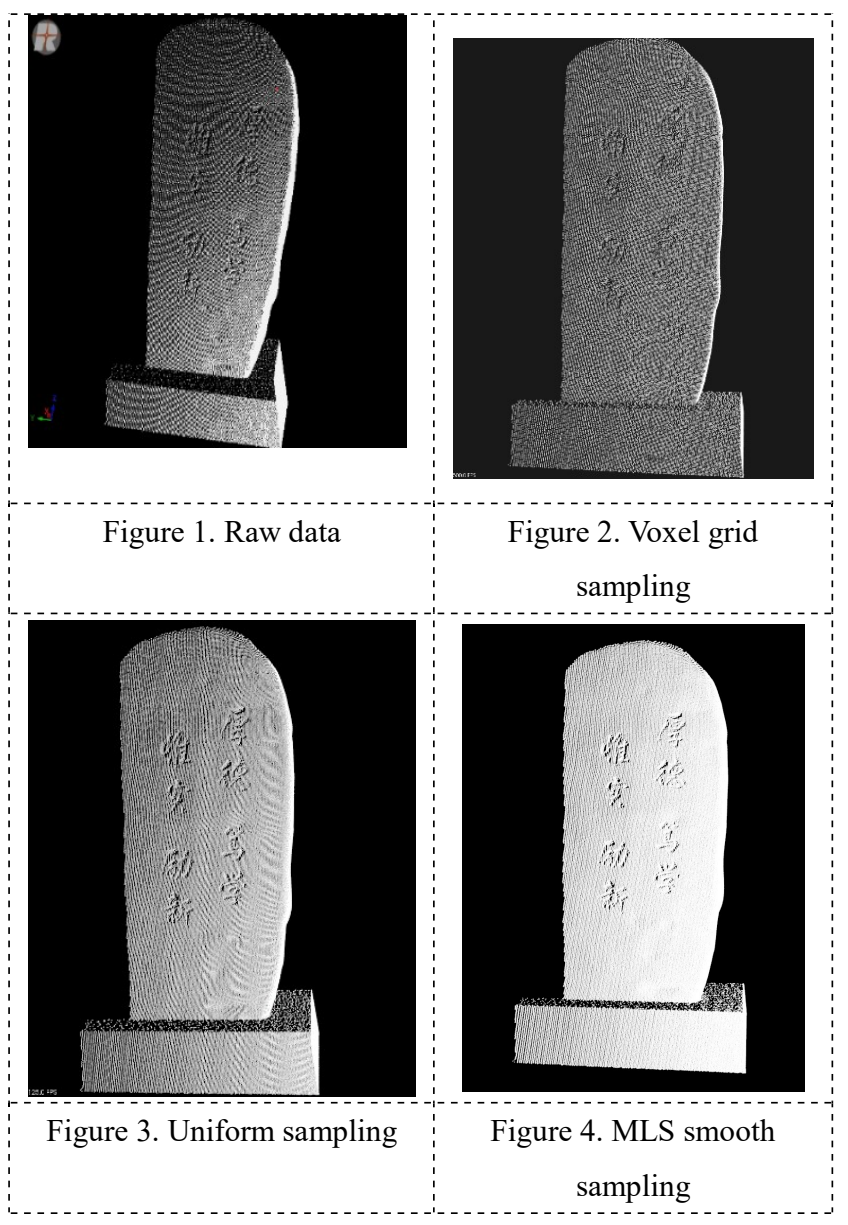

It can be seen from the comparison between Fig. 2 and Fig. 1 that the voxel grid sampling can well sample the point cloud data, reducing the amount of data, but the point cloud data detail feature points are also reduced. It can be seen from the comparison between Fig. 3 and Fig. 1 that uniform sampling can also reduce the amount of point cloud data, and the detailed feature point cloud can also be reflected, but the detail feature points are still reduced, and the good sampling effect is not achieved. It can be seen from the comparison between Fig. 4 and Fig. 1 that the amount of point cloud data is not decreased but the number of detail features is also increased, and the point cloud increases the smoothing effect. Compared to the previous two sampling directions, the overall point cloud data details look more prominent.

In order to further determine the influence of the search neighborhood radius on the detail effect of the value-added point cloud, the experiment also sets different search neighborhood radii for point cloud smooth sampling. As shown in Figure 5, the left half is the original data point cloud feature detail display, and the right half is the use algorithm. When the neighborhood radius $r=0.02$, the feature details of the right half are clearly displayed, and the point cloud of the detail feature is strengthened. When the neighborhood radius is $r=0.05$, the point cloud value-added is generally in the whole point set. Compared with the original data, the detailed feature points are more prominent, as shown in Fig. 6. It can be seen that as the radius of the search neighborhood increases, the amount of point cloud increment increases, but the longer the time is used, and the range of value increases will be reduced.

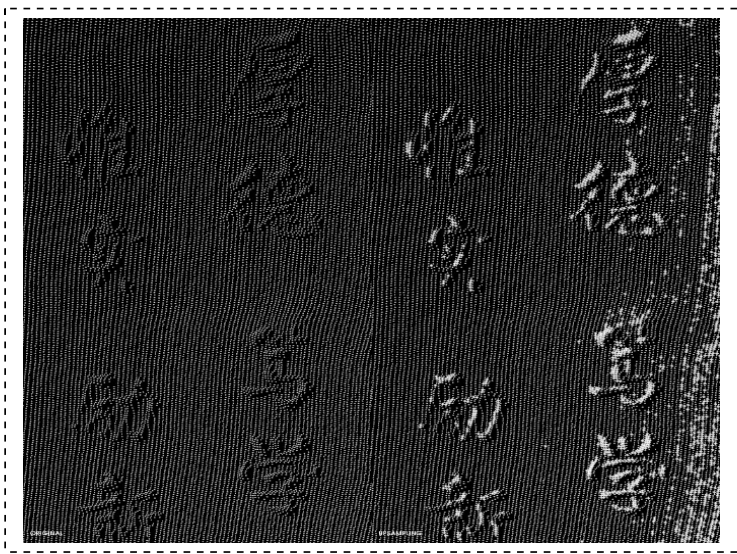

Figure 5. Search neighborhood radius $\mathrm{r}=0.02$

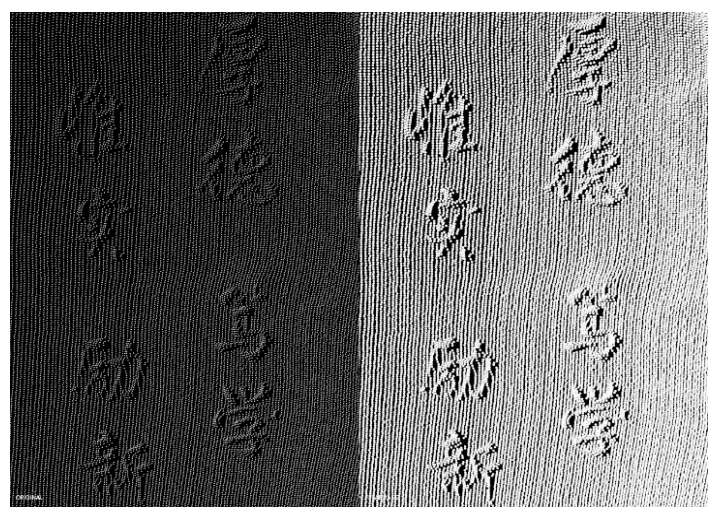

Figure 6. Search neighborhood radius $\mathrm{r}=0.05$

The surface of the above sampled experimental data was reconstructed using greedy projection triangulation. The experimental results are shown in Figure 7-10. It can be clearly seen from the comparison of Fig. 7(a), Fig. 8(a), Fig. 9(a) and Fig. 10(a) that Figure 7 (a), Figure 8 (a), Figure 9 (a) the surface of the model is rough, and there are holes in the surface. It can be clearly seen from the comparison of Fig. 7(b), Fig. 8(b), Fig. 9(b) and Fig. 10(b) that, the feature details of Figure 10(b) are relatively smooth, and the triangular faces that make up the model are not so obvious, while the triangular faces of the model can be clearly seen in Figure 7(b), Figure 8(b), and Figure 9(b). Figure 8(b) and Figure 9(b) due to the reduction of the feature detail point cloud during sampling, resulting in 
unclear defects in the detail features after reconstruction. It is shown that the model reconstructed by the point cloud smooth sampling data using the moving least squares method is better than the model reconstructed by the voxel grid sampling and the uniform sampling data.

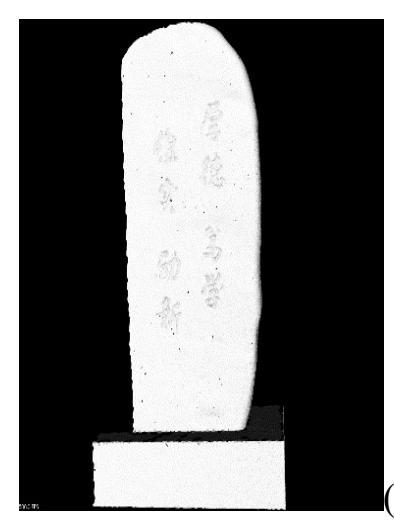

a) Raw data reconstruction model

Figure 7. Raw data reconstruction model and feature detail

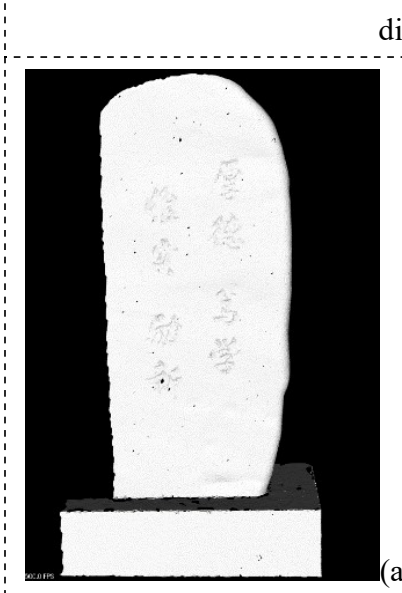

) Voxel grid sampling data reconstruction model

Figure 8. Voxel grid sampling data reconstruction model and feature detail display

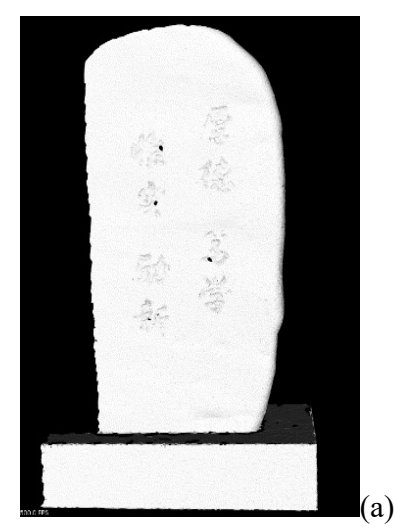

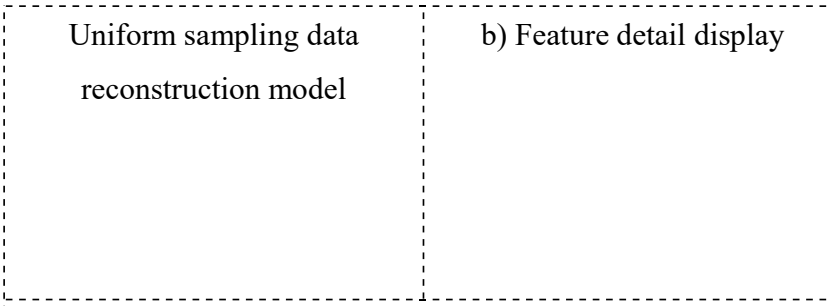

Figure 9. Uniformly sampled data reconstruction model and feature detail display
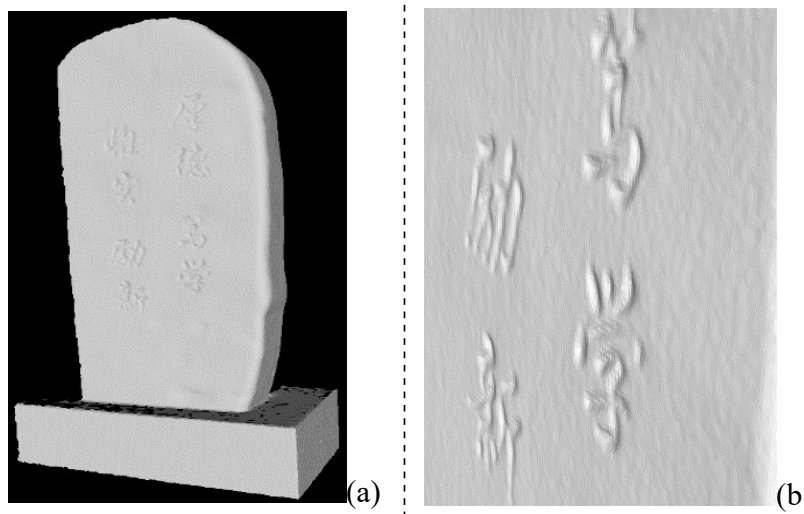

MLS smooth sampling data reconstruction model

) Feature detail display

Figure 10. MLS smooth sampled data reconstruction model and feature detail display

\section{CONCLUSION}

Point cloud data sampling and surface reconstruction are important aspects of point cloud data processing. In this paper, based on the current point cloud sampling method, the point cloud distribution is not uniform, the point cloud feature information is incomplete, and the reconstructed model surface is not smooth. A point cloud smooth sampling process and surface reconstruction using moving least squares method are proposed. The method first uses the moving least squares method for point cloud smoothing, and then uses the improved moving least squares method to add value, draws a circle centered on the sample point, and within a certain neighborhood radius, meets the value-added condition, then iteratively increases the point cloud, reduces the fitting deviation, and achieves a better fitting effect. Finally, the greedy projection method is used to reconstruct the surface of the sampled data. The experimental results show that after using the improved moving least squares method for point cloud smooth sampling, the point cloud fitting effect is better, the feature details point cloud is prominent, the reconstructed model is smoother, and the feature details are well preserved. 


\section{ACKNOWLEDGEMENT}

This paper is financially supported by the Guangxi Innovative Development Grand Grant under the grant number: Guike AA1-

8242048; Guilin Science and Technology Research and Development Projectthe grant number: 20170220; the "Ba Gui Scholars" program of The provincial government of Guangxi;and the Guangxi Key Laboratory of Spatial Information and Geomatics numbers 140452410,163802501.

\section{REFERENCES}

Bernard, F., Salamanca, L., Thunberg, J., 2017. Shape-aware surface reconstruction from sparse 3D point clouds. Medical Image Analysis, 2(5), 77-89.

Chen, Y., 2011. Research on algorithm related to scattered point cloud preprocessing in reverse engineering. University of Science and Technology of China.

Chen, J., Xia, C., Ying, H., 2013. Using facial symmetry in the illumination cone based $3 \mathrm{D}$ face reconstruction. IEEE International Conference on Image Processing, 3700-3704.

Guo, C., 2016. Fast algorithm for moving least squares method and its application. Chongqing University.

Huang, X., Yang, J., Chen, H., 2018. A 3D Laser Point Cloud Data Reduction Algorithm. Journal of Jishou University (Natural Science Edition), 39(05), 19-23.

Huang, X., Tian, Q., Mao, J., 2010. Adaptive moving least squares for scattering points fitting. WSEAS Transactions on Computers, 9(6), 664-673.

Kang, L., 2018. Research on the accuracy of free-form surface machining based on three-dimensional reconstruction of discrete point cloud. South China University of Technology.

Liang, Y., 2018. Research on Environment Modeling of Teleoperation Robot Based on Virtual Reality Technology. Digital Communication World, 12, 83-84.

Li, Z., 2016. Research on NURBS Surface Reconstruction
Based on Resampling. Nanchang University.

Ma, D., Li, G., Cui, J., 2017. Research on redundant data reduction algorithm of laser scanning point cloud based on curvature and normal. Boletín Técnico, 55(6), 273-278.

Shi, T., 2017. Research on 3D rail surface defect detection technology based on 2D/3D composite machine vision. Wuhan University of Science and Technology.

Tang, Z., Gao, B., Dou, M., 2019. Point cloud reduction algorithm based on weighted least squares curvature calculation Computer Engineering and Design, 40(06), 1606-1610+1659.

Wang, S., 2018. Design and implementation of 3D reconfigurable stored grain pest monitoring system based on virtual reality technology. Beijing University of Posts and Telecommunications.

Zhu, W., Li, X., Wei, J., 2017. Reconstruction of 3D model based on reverse engineering. PLASTICS SCIENCE, 45(04), 79-83.

Zhai, J., 2015. Research on spatial deconstruction point cloud denoising and thin reconstruction algorithm. Xi'an University of Science and Technology.

Zeng, Q., Lu, D., 2004. Curve and Surface Fitting Based on Moving Least Squares Method. Journal of Engineering Graphics, 1, 84-89. 